\title{
Mechanical Thrombectomy in Acute Stroke: Prospective Pilot Trial of the Solitaire FR Device while Under Conscious Sedation
}

\author{
S. Soize, K. Kadziolka, L. Estrade, I. Serre, S. Bakchine, and L. Pierot
}

\begin{abstract}
BACKGROUND AND PURPOSE: The best approach between general anesthesia and conscious sedation to perform mechanical thrombectomy remains unknown. The goal of our study was to evaluate the feasibility, safety, and efficacy of mechanical thrombectomy under conscious sedation in patients with acute ischemic stroke, using the Solitaire FR device, in a prospective, single-center, single-arm study.
\end{abstract}

MATERIALS AND METHODS: The study included consecutive patients with acute ischemic stroke due to a large artery occlusion within 6 hours of symptom onset for the anterior circulation, and within 24 hours for the posterior circulation. After intravenous thrombolysis (when no contraindications), thrombectomy was performed with the Solitaire device in patients under conscious sedation. Primary efficacy and safety end points were good functional outcome ( $m R S \leq 2)$ at 3 months and mortality at 3 months. Secondary end points were recanalization $(\mathrm{TICl} \geq 2)$ and failure rate.

RESULTS: From May 2010 to July 2011, 36 patients were treated. Median baseline NIHSS score was 17.5. The occlusion site was MCA in 21 patients (58.4\%), ICA-MCA tandem occlusion in 9 patients (25.0\%), terminal ICA in 2 patients (5.5\%), and basilar artery in 4 patients (11.1\%). Twenty-three patients $(63.9 \%)$ received intravenous thrombolysis. Superselective catheterization of the occluded vessel was not feasible in $5 / 36$ cases (13.9\%). Successful revascularization was achieved in 28/36 patients (77.8\%). After 3 months, 22 patients (61.1\%) showed good functional outcome (mRS $\leq 2)$ and the median NIHSS score was 8.5. Overall mortality rate at 3 months was $22.2 \%(8 / 36)$.

CONCLUSIONS: In acute ischemic stroke, mechanical thrombectomy while under conscious sedation is feasible in a large percentage of cases (86.1\%) and is associated with a short procedure delay and a high percentage of good functional outcomes at 3 months (61.1\%).

ABBREVIATIONS: ASPECT = Alberta Stroke Program Early CT; IQR = interquartile range; $\mathrm{mRS}=$ modified Rankin Scale; $\mathrm{TICl}=$ Thrombolysis in Cerebral Infarction

$\mathbf{P}$ atients presenting with acute ischemic stroke can be treated medically by lysing the clot via intravenous fibrinolysis or endovascularly by removing the clot through an intra-arterial method to re-establish vascularization in the blocked territory. The therapeutic reference for acute ischemic stroke is intravenous thrombolysis with rtPA within 4.5 hours of stroke onset. ${ }^{1,2}$ When the occluded artery is proximal or large, intravenous fibrinolysis

Received February 27, 2012; accepted after revision April 10.

From the Service de Radiologie (S.S., K.K., L.E., L.P.) and Service de Neurologie (I.S., S.B.), Hôpital Maison Blanche, Université de Reims, Reims, France.

Contributors to the paper: Sébastien Soize: study concept and design, definition of intellectual content, literature research, data analysis, manuscript preparation, and manuscript editing; Krzysztof Kadziolka: data acquisition and manuscript review; Laurent Estrade: data acquisition and manuscript review; Isabelle Serre: data acquisition and manuscript review; Serge Bakchine: manuscript review; Laurent Pierot: study concept and design, definition of intellectual content, literature research, manuscript preparation, manuscript editing, and manuscript review.

Please address correspondence to Laurent Pierot, MD, PhD, Department of Radiology, Maison Blanche Hospital, 45 Rue Cognacq Jay, 51092 Reims, France; e-mail: Ipierot@gmail.com

http://dx.doi.org/10.3174/ajnr.A3200 often reaches its limits, resulting in low recanalization rates and a high degree of reocclusion. ${ }^{3,4}$ Because early recanalization is highly correlated with a patient's prognosis, various endovascular techniques have been developed to improve the recanalization rate as well as the clinical outcome of these patients. ${ }^{5}$ The Prolyse in Acute Cerebral Thromboembolism (PROACT) II study demonstrated the efficacy of intra-arterial fibrinolysis with a high recanalization rate of $66 \%$ in the MCA. ${ }^{6}$ Then, studies dealing with various mechanical thrombectomy devices demonstrated increased recanalization rates, ranging from 59\% to $90 \%$, and improved clinical outcome..$^{7-12}$

Intra-arterial thrombectomy was initially performed under general anesthesia, but recent publications have outlined the potential value of conscious sedation with improved clinical outcomes after 3 months and a lower mortality rate. ${ }^{13-16}$ However, these studies have several limitations: first, they were retrospective and the feasibility of mechanical thrombectomy under conscious sedation was not analyzable; second, only some occlusion sites were included (anterior circulation or middle cerebral artery); 
third, some patients were treated under conscious sedation and some under general anesthesia, introducing a selection bias; and fourth, patients were treated with chemical thrombolysis or mechanical thrombolysis using first-generation devices (eg, Merci [Concentric Medical, Mountain View, California], Penumbra [Penumbra, Alameda, California]).

Retrievable stents are among the most promising devices used for mechanical thrombectomy. The Solitaire FR device (ev3, Irvine, California) was evaluated in small retrospective series of patients usually treated under general anesthesia. ${ }^{9-12}$ However, the results of mechanical thrombectomy using the Solitaire device under conscious sedation are not actually known.

\section{MATERIALS AND METHODS}

The goal of this pilot study was to prospectively evaluate the feasibility, safety, and efficacy of mechanical thrombectomy using the Solitaire FR device in patients with acute ischemic stroke treated under conscious sedation. We compared our results with the other Solitaire series of patients under general anesthesia.

The protocol for this study received institutional review board approval. Informed consent was obtained from the patients or their relatives.

This was a prospective single-center, single-arm, intention-totreat study. All patients with acute stroke with relevant neurologic symptoms, as defined by an NIHSS score $\geq 8$, presenting within 6 hours of symptom onset for the anterior circulation and within 24 hours for the posterior circulation, with occlusion of a large intracranial vessel (ICA and/or MCA; basilar artery) depicted on MR imaging (or CT angiography when contraindication) and confirmed by angiography (TICI grade 0 or 1 ), and without initial spontaneous intracranial hemorrhage were consecutively included. The exclusion criteria were spontaneous NIHSS improvement, recanalization before endovascular treatment viewed on the first angiographic run, and contraindication to endovascular treatment (vascular blush, fistula) determined on the first-run angiogram.

Upon admission, each patient with suspected acute ischemic stroke was examined by an experienced stroke unit neurologist. An acute MR imaging was then performed. Blood pressure, pulse, and respiration rate were monitored continuously and watched by the stroke unit neurologists. The NIHSS score was calculated to determine a clinical severity score. The stroke unit neurologist administered IV tPA (alteplase [Actilyse]; Boehringer, Ingelheim, France) at an effective dose $(0.9 \mathrm{mg} / \mathrm{kg})$ within a 4.5 -hour window, if no contraindication. The interventional radiologist on call was notified, and when the diagnosis was confirmed, the patient was transferred to the angiography suite (immediately for patients who did not receive intravenous thrombolysis, or after 30-60 minutes with no clinical improvement for those who received it). Because we did not have an anesthesia team dedicated to this procedure, the stroke neurologist provided conscious sedation. All patients received $1 \mathrm{mg}$ of IV midazolam before the arterial puncture. Then, in case of agitation or pain, an IV bolus of $1 \mathrm{mg}$ of midazolam was administered and repeated, if necessary. Standby intubation was not available during the procedures, as anesthesiologists were involved only in severe cases. Blood pressure was lowered with nicardipine in case of uncompensated hypertension, defined as systolic blood pressure exceeding $180 \mathrm{~mm} \mathrm{Hg}$ or diastolic blood pressure exceeding $110 \mathrm{~mm} \mathrm{Hg}$. After the endovascular treatment, the patient was transferred to the stroke intensive care unit.

An acute 3T MR imaging (Achieva 2.1; Philips Healthcare, Best, the Netherlands) was performed to confirm the diagnosis of stroke, locate the occluded vessel, and search for exclusion criteria. DWI was used to assess the extent of the ischemic lesion. A time-of-flight sequence focused on the circle of Willis was used to locate the site of vessel occlusion. T2 gradient-echo imaging was performed to screen for intracranial hemorrhage. Flair-weighted imaging completed the protocol. No perfusion-weighted imaging was acquired. We calculated the ASPECT score on DWI to assess the ischemic severity score for the anterior circulation and pcASPECT for posterior circulation. ${ }^{17,18}$ In case of MR imaging contraindication, a head CT and CT angiography were performed to confirm the vessel occlusion. The occlusion location was divided into 4 categories: MCA including M1, M1-M2, and M2; ICA; basilar artery; and ICA-MCA tandem occlusion (occlusion of the proximal ICA and MCA).

Three neurointerventionalists performed the mechanical thrombectomy on a biplanar system (Axiom Artis dBA; Siemens, Erlangen, Germany). All patients were treated via a femoral arterial approach while under conscious sedation. In either the anterior or posterior circulation, we used a $6 \mathrm{~F}$ guide catheter (Chaperon 6F; MicroVention, Aliso Viejo, California) or a 6F long sheath (Flexor Tuohy-Borst; Cook, Bloomington, Indiana) to reach the target artery. No intravenous heparin was administered if an intravenous thrombolysis was performed before the mechanical thrombectomy. If not, $2500 \mathrm{IU}$ of heparin was delivered at this time. Occlusion of the target vessel was verified angiographically and rated on the TICI scale. Using a coaxial system, we advanced a 0.21-inch internal diameter microcatheter (Prowler Select Plus; Cordis, Miami Lakes, Florida, or Rebar -027; ev3) over a guidewire (Tracker Excel 14; Boston Scientific, Natick, Massachusetts) placed through the thrombus. After removal of the guidewire, a microcatheter angiographic run was performed to confirm the correct positioning and visualize the vasculature distal to the thrombus. All patients were treated using the Solitaire FR device (ev3). Under fluoroscopic control, the Solitaire was advanced through the microcatheter across the vessel occlusion and deployed completely by pulling back the microcatheter. An angiographic run was performed to evaluate the correct placement and expansion of the device and control for flow restoration. The stent was kept deployed for 3-5 minutes before retrieval. We did not use a balloon guide catheter to achieve flow arrest, nor did we work with a special aspiration catheter. The unsheathed Solitaire stent and microcatheter were then removed (as a single unit) into the guide catheter, while applying suction through the guide catheter using a 20-mL syringe (VacLok Syringe; Merit Medical, South Jordan, Utah). An angiographic run was performed to evaluate flow restoration. If necessary, the entire process was repeated until the end of the therapeutic window. Groin punctures were routinely closed with an Angio-Seal (St. Jude Medical, Minnetonka, Minnesota). In cases of tandem occlusion, the choice of whether to treat the ICA or MCA occlusion first was decided by the neurointerventionalist, considering technical con- 
ditions (tortuous vessel), remaining time to treat, and extent of the lesions. The interruption of the procedure before complete recanalization was decided on a case-by-case basis by the interventional neuroradiologist and the neurologist, according to remaining time to treat, the clinical status of the patient (growing agitation), and vascular anatomy (very atheromatous vessels), avoiding any technical complications or clinical worsening.

Clinical data were recorded for every patient upon admission, at the end of the endovascular procedure, at day 1 , at discharge by the neurologists in the stroke unit, and at the 3-month consultation conducted by an independent neurologist. When a face-toface visit was impossible, the independent neurologist performed the Rankin assessment by phone.

The following time points were collected for every patient: time from symptom onset to the beginning of the endovascular procedure (defined as the first angiographic run); duration of the procedure (from the first to the last angiographic run); and time from symptom onset to recanalization (last angiographic run). Revascularization was assessed on a final angiographic run performed immediately after the end of the procedure. Flow restoration was evaluated by the neurointerventionalist using the TICI flow classification. Symptomatic hemorrhagic events were evaluated using a 24-hour CT. A symptomatic hemorrhagic event was defined as any intracerebral bleeding causing neurologic deterioration (NIHSS increase $\geq 4$ ).

Schematically, we had 3 categories of results: "recanalized" if TICI score $\geq 2 \mathrm{a}$, "treatment failure" if we were unable to attempt a recanalization (the stent was not opened), and "no recanalization before exceeded deadline." The efficacy and safety criteria were evaluated in the whole series and defined according to the trial design and reporting standards for intra-arterial cerebral thrombolysis for acute ischemic stroke suggested by Higashida et al. ${ }^{19}$ The main efficacy judgment criterion was functional outcome at 3 months, defined by an $\mathrm{mRS} \leq 2$. Recanalization, defined by the final TICI scale on the last angiographic run, and neurologic score, according to NIHSS criteria at 3 months, were secondary outcomes. The main safety outcome was mortality at 3 months. Symptomatic hemorrhage and procedural-linked or procedural-unlinked complication rates were also reported.

The patient characteristics and results were represented by extreme values, mean and standard deviation, median and interquartile range for continuous variables, and by frequencies and proportions for categoric variables. Statistical analysis was performed using Openstat 17.08.10 software (Softonic, Barcelona, Spain).

\section{RESULTS}

From May 2010 to August 2011, 36 patients aged 15-85 years $($ mean $=64.0 \pm 16.5$ years; median $=69.5[\mathrm{IQR}=56-75])$ presenting with an acute ischemic stroke with a large vessel occlusion were treated at our institution with the Solitaire stent (Table 1). NIHSS score upon admission was $8-35($ mean $=17.1 \pm 6.6)$ with a median of $17.5(\mathrm{IQR}=12.5-21)$. Twenty-three patients (63.9\%) had no contraindication to intravenous thrombolysis and received tissue plasminogen activator before the neurointerventional approach, and 13 patients $(36.1 \%)$ had contraindications for fibrinolysis (exceeded the deadline and recent extracra-

\section{Table 1: Patient characteristics}

\begin{tabular}{ll}
\hline Age in years (median) (IQR) & $69.5(56-75)$ \\
Female (No.) (\%) & $18(50 \%)$ \\
Baseline NIHSS score (median) (IQR) & $17.5(12.5-21)$ \\
Site of occlusion (No.) (\%) & \\
MCA & $21(58.4 \%)$ \\
Terminal ICA & $2(5.5 \%)$ \\
Tandem occlusion & $9(25.0 \%)$ \\
Basilar artery & $4(11.1 \%)$ \\
Combined therapy with rtPA (No.) (\%) & $23(63.9 \%)$ \\
ASPECT score on DWI (median) (IQR) & $7(5-8)$ \\
Anterior circulation ( $n=32)$ & $7(5-8)$ \\
Posterior circulation $(n=4)$ & $8(8-8.5)$ \\
Baseline TICI 0/1 (No.) (\%) & $31(86.1 \%) / 5(13.9 \%)$ \\
\hline
\end{tabular}

Note:-Tandem occlusion indicates occlusion of both ICA and MCA.

nial bleeds). The occluded vessel was the MCA in 21 patients (58.4\%), the basilar artery in 4 patients $(11.1 \%)$, the terminus ICA in 2 patients (5.4\%), and both the cervical carotid artery and the carotid bifurcation or MCA (tandem occlusion) in 9 patients (25.0\%). ASPECT score on DWI was 3-9 (mean = $6.6 \pm 1.9$; median $=7[\mathrm{IQR}=5-8])$. On the initial angiogram, all patients had a complete vessel occlusion defined as a TICI score of 0 ( $n=$ $31,86.1 \%)$ or $1(n=5,13.9 \%)$. Because the study was based on an intention-to-treat population, we included 1 patient $(2.7 \%)$ who required intubation before the endovascular procedure because of rapid alteration of the level of consciousness.

We were not able to attempt a recanalization in the therapeutic window for 5 patients. Time was lost due to patient agitation in 3 cases $(8.3 \%)$ and due to significant tortuosity of the cervical vessels in 2 cases (5.6\%). Finally, the failure rate of treatment was $13.9 \%$. As a consequence, only 31 patients were treated: 28 were recanalized and 3 were not recanalized in the time remaining (despite 1 or 2 openings of the stent).

Regarding the 3 agitated patients for whom the treatment failed, the remaining time was too short to have them under general anesthesia and continue the treatment by mechanical thrombectomy. In the 2 patients untreated due to vessel tortuosity, 1 had a good $(m R S=2)$ and 1 had a moderate $(m R S=3)$ functional outcome at 3 months.

Seven patients were agitated and needed addition of midazolam during the procedure (from 2-13 mg). After 3 months, 3 of these 7 patients presented with a good functional outcome (mRS $\leq 2), 2$ had a moderate or bad functional outcome ( $\mathrm{mRS}=4$ and 5 ), and 2 patients died of symptomatic intracranial hemorrhage. The 3 patients with failed treatment had an $\mathrm{mRS}$ of 2 , an $\mathrm{mRS}$ of 5 , and 1 was deceased at 3 months.

Time from symptom onset to endovascular treatment was 115-360 minutes $($ mean $=269 \pm 60$ minutes; median $=275$ minutes $[\mathrm{IQR}=225-320])$. The duration of the procedure was $12-81$ minutes $($ mean $=33 \pm 20$ minutes; median $=27.5 \mathrm{~min}$ utes $[\mathrm{IQR}=20-46]$ ). The time from symptom onset to recanalization was 122-405 minutes (mean $=302 \pm 65$ minutes; median $=320$ minutes $[\mathrm{IQR}=256-48])$. Successful revascularization was achieved in 28 of $36(77.8 \%)$ patients (TICI $\geq 2)$ ( Table 2). TICI 3 was achieved in 14 patients $(38.9 \%)$ and TICI $2 \mathrm{~b}$ occurred in 6 patients $(16.7 \%)$. The median number of stent deployments was $1.5(\mathrm{IQR}=1-2)$. In 2 patients $(5.5 \%)$, ICA stent placement (Wallstent; Boston Scientific) was necessary to access the target vessel because of acute occlusion of the cervical ICA. 
Table 2: Safety: mortality and procedural complications

\begin{tabular}{lc}
\hline & $\boldsymbol{n}(\%)$ \\
\hline Mortality at 3 months & $8(22.2 \%)$ \\
Symptomatic ICH & $5(13.9 \%)$ \\
Cerebral edema & $3(8.3 \%)$ \\
Failure rate & $5(13.9 \%)$ \\
Device-related complications (dissection) & $1(2.8 \%)$ \\
Symptomatic ICH at 24 hours & $3(8.3 \%)$ \\
Symptomatic ICH at 3 months & $6(16.7 \%)$ \\
Fatal outcomes & $5(13.9 \%)$ \\
Poor outcome (mRS 5) & $1(2.8 \%)$ \\
\hline
\end{tabular}

Note:- $-\mathrm{ICH}$ indicates intracranial hemorrhage.

At 3 months, 22 patients (61.1\%) showed good functional outcome (mRS $\leq 2), 4$ patients $(11.1 \%)$ had a moderate outcome $(\mathrm{mRS}=3$ or 4$)$, and 10 patients $(27.8 \%)$ had a poor outcome $(\mathrm{mRS}=5)$ or died. At 3 months, median NIHSS score (calculated on the 28 living patients) was 8.5 (IQR $=1-14$ ), with a mean $( \pm S D)$ of $9.0(8.8)$. Eighteen patients $(50 \%)$ presented with a NIHSS score of $\leq 1$ or an improvement of at least 8 points from baseline.

Hemorrhagic complications were depicted on 24-hour CT in 10 patients $(27.7 \%)-7$ were asymptomatic (19.4\%) and 3 were symptomatic $(8.3 \%)$. Symptomatic hemorrhage was observed in 1 patient with tandem occlusion and agitation during the treatment, 1 patient with tandem occlusion previously treated with IV thrombolysis and no agitation, and 1 patient with MCA occlusion. Seven of 10 patients $(70 \%)$ had combined treatment with intravenous thrombolysis. Symptomatic hemorrhagic complications at 3 months concerned 6 patients (16.7\%), 3 already diagnosed at 24 hours and 3 occuring, respectively, at day 3 , day 8 , and day 8 .

The overall mortality rate at 3 months was $22.2 \%(8 / 36)$ (Table $3)$. There were 5 fatal outcomes $(13.9 \%)$ due to symptomatic intracranial hemorrhage, and 3 patients died of extensive brain infarction with intracranial hypertension (their recanalization status was, respectively, TICI 0,1 , and $2 \mathrm{a}$ ).

We observed $1(2.7 \%)$ arterial dissection of segment M1 of the MCA in a nonagitated patient with extravasation of contrast media during the procedure, and hematoma on the postoperative CT. No functional improvement was observed at 3 months.
Table 3: Efficacy: angiographic and clinical outcome

Time from stroke symptom onset to recanalization (min) (median) (IQR)

Anterior circulation $(n=32)$

Posterior circulation $(n=4)$

$320(256-344)$

$331(267-371)$

$27.5(20-46)$

$28(77.8 \%)$

Recanalization rate ${ }^{\mathrm{b}}$ (No.) (\%)

$\mathrm{TICl}$

3

$2 b$

$2 a$

1

0

$14(38.9 \%)$

$6(16.7 \%)$

$8(22.2 \%)$

$2(5.5 \%)$

$6(16.7 \%)$

$1.5(1-2)$

$8.5(1-14)$

$22(61.1 \%)$

NIHSS score at discharge ${ }^{c}$ (min) (median) (IQR)

mRS $<2$ at 3 months (No.) (\%)

Note:-min indicates minutes.

a From the first angiogram to recanalization.

${ }^{b}$ Defined by $\mathrm{TICl} \geq 2 \mathrm{a}$.

${ }^{c}$ Calculated in living patients $(n=28)$.

\section{DISCUSSION}

Various types of sedation are used in acute ischemic stroke interventions. The operator's habits and the institution's opportunity to have an anesthetic support team are the main factors for the decision to treat while under general anesthesia or conscious sedation. Three recent reports of retrospective data suggest that general anesthesia may negatively impact the outcome of acute ischemic stroke interventions. ${ }^{14-16}$ Two series recently demonstrated that chemical (intra-arterial urokinase) and mechanical thrombectomy (using Merci and Penumbra devices) under conscious sedation were as safe as under general anesthesia and provided a better clinical outcome after 3 months (mRS $\leq 2) .{ }^{14,15}$ Abou-Chebl et al also noticed a lower mortality rate with conscious sedation. ${ }^{14}$ However, these 2 large studies had several limitations, including their retrospective nature, selection bias, and inclusion of some occlusion locations only.

Potential disadvantages of general anesthesia are increased delay in time to recanalization due to intubation and ventilation, hypotension with administration of induction agents, uncontrolled hypertension during intubation, and prolonged intubation after the procedure leading to longer intensive care unit stays. These disadvantages are overcome by conscious sedation, which

Table 4: Comparison with other Solitaire patient series

\begin{tabular}{|c|c|c|c|c|c|}
\hline & Present Study & Machi et al ${ }^{11}$ & Roth et $\mathrm{al}^{10}$ & Castaño et $a^{9 a}$ & Stampfl et al ${ }^{12}$ \\
\hline Number of patients & 36 & 56 & 22 & 20 & 18 \\
\hline CS or GA & CS & GA & Both & GA & GA \\
\hline Baseline NIHSS score & 17.1 & 15.8 & 19.4 & 19 & 21 \\
\hline ASPECT score & 6.6 & - & - & - & - \\
\hline Symptom onset to recanalization (min) & 302 & 353 & 277 & 352 & 289 \\
\hline Successful recanalization & $77.8 \%{ }^{c}$ & $89 \%{ }^{d}$ & $90.9 \%{ }^{c}$ & $90 \%{ }^{d}$ & $88 \%{ }^{c}$ \\
\hline NIHSS score at discharge & 9 & 7 & - & 9.4 & - \\
\hline Symptomatic $\mathrm{ICH}^{\mathrm{b}}$ & $8.3 \%$ & $1.7 \%$ & $13.6 \%$ & $10 \%{ }^{\mathrm{e}}$ & $16.6 \%$ \\
\hline $\mathrm{mRS} \leq 2$ at 3 months & $61.1 \%$ & $46 \%$ & $50 \%$ & $45 \%$ & $33.30 \%{ }^{e}$ \\
\hline Mortality at 3 months & $22.2 \%$ & $7.1 \%{ }^{\mathrm{e}}$ & $18.1 \%$ & $20 \%$ & $33 \%^{\mathrm{e}}$ \\
\hline
\end{tabular}

Note:-We show our results with mean in order to favor comparison with other series. Only the Castaño et al ${ }^{9}$ results are presented with median. CS indicates conscious sedation; GA, general anesthesia; ICH, intracranial hemorrhage; min, minutes.

${ }^{a}$ In this series, the Solitaire device was not always used alone (3 times after the Merci device and twice after intra-arterial tissue plasminogen activator).

bepicted on CT (at 24 hours).

${ }^{c}$ Defined by $\mathrm{TICl} \geq 2 \mathrm{a}$.

${ }^{\mathrm{d}}$ Defined by $\mathrm{TICl} \geq 2 \mathrm{~b}$.

e Evaluation was done at discharge. 
also allows clinical evaluations of the patient during the procedure. On the other hand, conscious sedation can be associated with the lack of patient cooperation, potentially leading to intraprocedural complications.

At our center, the decision to treat under conscious sedation was initially due to the lack of an anesthesia team available for mechanical thrombectomy procedures. Our series was prospective, and the analyses were conducted in an intention-to-treat population. Therefore, precise knowledge of the feasibility, efficacy, and safety of mechanical thrombectomy using the Solitaire device under conscious sedation can be obtained.

In our series, mechanical thrombectomy under conscious sedation was feasible in a high percentage of cases $(86.1 \%)$. Treatment failed in 5 cases $(13.9 \%)$ due to anesthetic conditions (patient agitation) in 3 cases ( $8.3 \%$ ), and significant vessel tortuosity in 2 cases $(5.6 \%)$. In addition, in 1 case, rapid worsening of the clinical condition led to intubation and general anesthesia at the beginning of the endovascular procedure. Because our analysis was conducted on an intention-to-treat basis, these patients were included in the recanalization rate determination and safety and functional outcomes at 3 months.

The recanalization rate parameter is not as easy to handle because it can be evaluated in different ways from 1 series to another. The recanalization rate is certainly improved if a final angiographic run is performed with some delay after the end of the procedure. In addition, the differentiation between TICI $2 \mathrm{a}$ and $2 \mathrm{~b}$ is not always easy to assess. In our series, the final angiographic run was performed immediately after the last Solitaire pass and a strict TICI score grading was used.

Because of the failed procedures, the recanalization rate (77.8\%) was lower compared with other Solitaire series (89\% and $90.9 \%$ in the 2 largest series). ${ }^{10,11}$ Excluding patients with failed treatment, the recanalization rate would be very similar to those observed in other series (28/31 patients; $90.3 \%$ ). In addition, the recanalization rate in our series compares favorably with those in the MERCI and Penumbra trials $(69.5 \%$ and $81.6 \%$, respectively). ${ }^{7,8}$

Despite the relatively low recanalization rate observed in our series, a higher percentage of patients were functionally independent after 3 months (61.1\%) compared with the Solitaire series under general anesthesia (50\%). ${ }^{10}$ One potential explanation was a shorter time to recanalization compared with other Solitaire series (Table 4).

Overall mortality at 3 months was $22.2 \%$, which was in agreement with rates reported from other series, ranging from $7.1 \%$ (evaluation at discharge) for Machi et $\mathrm{al}^{11}$ to $33 \%$ for Stampfl et al. ${ }^{12}$ Symptomatic hemorrhagic complications at 24 hours occurred in $3 / 36(8.3 \%)$, which equals other series, ranging from $1.7 \%$ for the Machi et al series to $16.6 \%$ for Stampfl et al. ${ }^{9,11,12}$ One case of arterial dissection of the M1 part of the MCA $(2.7 \%)$ was observed in our series, leading to symptomatic intracranial hemorrhage with a poor 3-month clinical outcome $(\mathrm{mRS}=5)$. This complication was not previously reported in Solitaire series. It was probably related to inappropriate manipulations of the guidewire to pass through the clot in a patient who was not agitated.

Our study had several limitations. First, the number of pa- tients was relatively small, but the sample size is similar to other previously published Solitaire series. Second, the lack of a control group, which was the main flaw of any of those series (including ours), made it difficult to judge the clinical relevance of this treatment.

\section{CONCLUSIONS}

In acute ischemic stroke, mechanical thrombectomy while the patient is under conscious sedation is feasible in a high percentage of cases $(86.1 \%)$. Furthermore, a good functional outcome at 3 months was observed in a high percentage of cases $(61.1 \%)$. Consequently, in most cases, conscious sedation appears to be a valuable alternative to general anesthesia. Nevertheless, anesthesiologists will have a major place in stroke endovascular therapy by selecting and giving the most appropriate and least deleterious anesthesia to patients with acute stroke treated by mechanical thrombectomy. Further studies are needed to directly compare the outcome of patients treated with mechanical thrombectomy under general anesthesia or conscious sedation.

Disclosures: Laurent Pierot-UNRELATED: Consultancy: Codman, eV3, MicroVention, Penumbra, Sequent.

\section{REFERENCES}

1. Hacke W, Kaste M, Bluhmki E, et al. Thrombolysis with alteplase 3 to 4.5 hours after acute ischemic stroke. $N$ Engl $\mathrm{J}$ Med 2008;389:1317-29

2. Bluhmki E, Chamorro A, Dávalos A, et al. Stroke treatment with alteplase given $3.0-4.5 \mathrm{~h}$ after onset of acute ischaemic stroke (ECASS III): additional outcomes and subgroup analysis of a randomised controlled trial. Lancet Neurol 2009;8:1095-102

3. Del Zoppo GJ, Poeck K, Pessin MS, et al. Recombinant tissue plasminogen activator in acute thrombotic and embolic stroke. Ann Neurol 1992;32:78-86

4. Alexandrov AV, Grotta JC. Arterial reocclusion in stroke patients treated with intravenous tissue plasminogen activator. Neurology 2002;32:862-67

5. Khatri P, Abruzzo T, Yeatts SD, et al. IMS I and II Investigators. Good clinical outcome after ischemic stroke with revascularization is time-dependent. Neurology 2009;73:1066-72

6. Furlan A, Higashida R, Wechsler L, et al for the PROACT Investigators. Intra-arterial prourokinase for acute ischemic stroke. The PROACT II study: a randomized controlled trial. JAMA 1999;282:2003-11

7. Smith WD, Sung G, Saver J, et al. Mechanical thrombectomy for acute ischemic stroke: final results of the Multi MERCI trial. Stroke 2008;39:1205-12

8. The Penumbra Pivotal Stroke Trial Investigators. The Penumbra Pivotal Stroke Trial: safety and effectiveness of a new generation of mechanical devices for clot removal in intracranial large vessel occlusive disease. Stroke 2009;40:2761-68

9. Castaño C, Dorado L, Guerrero C, et al. Mechanical thrombectomy with the Solitaire $\mathrm{AB}$ device in large artery occlusions of the anterior circulation. A pilot study. Stroke 2010;41:1836-40

10. Roth C, Papanagiotou P, Behnke S, et al. Stent-assisted mechanical recanalization for treatment of acute intracerebral artery occlusions. Stroke 2010;41:2559-67

11. Machi P, Costalat V, Lobotesis K, et al. Solitaire FR thrombectomy system: immediate results in $\mathbf{5 6}$ consecutive acute ischemic stroke patients. J Neurointerv Surg 2012;4:62-66

12. Stampfl S, Hartmann M, Ringleb PA, et al. Stent placement for flow restoration in acute ischemic stroke: a single-center experience with the Solitaire stent system. AJNR Am J Neuroradiol 2011;32:1245-48 
13. McDonagh DL, Olson DM, Kalia JS, et al. Anesthesia and sedation practices among neurointerventionalists during acute ischemic stroke endovascular therapy. Front Neur 2010;1:118

14. Abou-Chebl A, Lin R, Hussain MS, et al. Conscious sedation versus general anesthesia during endovascular therapy for anterior circulation stroke: preliminary results from a retrospective, multicenter study. Stroke 2010;41:1175-79

15. Jumaa MA, Zhang F, Ruiz-Ares G, et al. Comparison of safety and clinical and radiographic outcomes in endovascular acute stroke therapy for proximal middle cerebral artery occlusion with intubation and general anesthesia versus the nonintubated state. Stroke 2010;41:1180-84
16. Nichols C, Carrozzella J, Yeatts $S$, et al. Is periprocedural sedation during acute stroke therapy associated with poorer functional outcomes? J NeuroIntervent Surg 2010;2:67-70

17. Pexman JH, Barber PA, Hill MD, et al. Use of the Alberta Stroke Program Early CT Score (ASPECTS) for assessing CT scans in patients with acute stroke. AJNR Am J Neuroradiol 2001;22:1534-42

18. Tei H, Uchiyama S, Usui T, et al. Posterior circulation ASPECTS on diffusion-weighted MRI can be a powerful marker for predicting functional outcome. J Neurol 2010;257:767-73

19. Higashida RT, Furlan AJ, Roberts H. Trial design and reporting standards intra-arterial cerebral thrombolysis for acute ischemic stroke. Stroke 2003;34:109-37 